\title{
Editorials
}

\section{Understanding hemodynamic responses to tracheal intubation}

Orlando Hung MD FRCPC

I $\mathrm{T}$ is well recognized that laryngoscopic tracheal intubation is associated with hypertension and tachycardia mediated by sympathetic responses. Many pharmacologic interventions have been suggested to attenuate these reflex responses to laryngoscopic intubation. These include the use of a variety of anesthetic agents, beta-blockers, vasodilators, local anesthetics including lidocaine, and magnesium. ${ }^{1-5}$ Unfortunately, none of these pharmacological manipulations can consistently and effectively attenuate these adverse responses, nor are they free from complications.

In a complex dynamic biological system, such as the human body, it is often difficult to appreciate the complicated pharmacokinetic-pharmacodynamic relationship of drug effects. The onset of drug effect among commonly used anesthetics varies significantly, depending on the physico-chemical characteristics of the drugs. In general, under a pharmacokinetic steady-state condition, concentration-effect relationships can be described by several relatively simple pharmacodynamic models. ${ }^{6}$ However, this concentration-effect relationship becomes more complex when a drug is administered under a non-steady state, such as a single bolus $i v$ administration. In the non steadystate paradigm, there is a time lag between changes in plasma concentrations of drugs and the corresponding observed drug effects, because of the blood-effect site equilibration time. Therefore, a more sophisticated and integrated pharmacokinetic-pharmacodynamic model is necessary to link and account for a possible temporal dissociation between the plasma concentration and the observed effect. ${ }^{7}$

Under non-steady state conditions, plasma and effect site (such as the brain in the case of anesthetics) concentrations change rapidly following drug admin- istration. Unless a high frequency measurement of drug effect is employed, such as a continuous recording of blood pressure or electroencephalogram, it may not be possible to fully characterize the true dynamic nature of the drug effect. In other words, it is possible that the peak drug effect will be missed.

The interpretation of cardiovascular responses to anesthetic drugs becomes even more difficult when a noxious intervention, such as a laryngoscopic intubation, introduces confounding variables. These include not only the magnitude and duration of the noxious intervention but also the variables introduced by various medical conditions, such as cardiovascular diseases and diabetes. This multitude of confounding variables may, in part, explain the inconsistent results reported in many previous studies.

In this issue, Takita and colleagues examined the efficacy of tracheal lidocaine in attenuating cardiovascular responses to endotracheal intubation following induction of anesthesia. ${ }^{8}$ Although topical lidocaine has been found to be ineffective in the past, ${ }^{9}$ Takita et al. took the appropriate approach of waiting for the onset of effect (two minutes) to allow topical anesthesia to take place before performing tracheal intubation. Cardiovascular responses in this group of patients were significantly less than that of the group with no tracheal lidocaine, as well as the group with tracheal intubation immediately following tracheal lidocaine. The authors concluded that tracheal lidocaine is effective in attenuating cardiovascular responses to laryngoscopic intubation, provided lidocaine is administered two minutes prior to tracheal intubation.

Unfortunately, the investigators did not employ high frequency effect measurement during the study to assess the onset of the pharmacodynamic effect of lidocaine. It is possible that the onset of peak drug

From the Department of Anaesthesia, Dalhousie University, Queen Elizabeth II Health Sciences Centre, Halifax, Nova Scotia, Canada. Address correspondence to: Dr. Orlando Hung, Department of Anaesthesia, Queen Elizabeth II Health Sciences Centre, Victoria General Site, 1278 Tower Road, Halifax, Nova Scotia B3H 2Y9, Canada. Phone: 902-473-7767; Fax: 902-423-9454; E-mail: hungorla@is.dal.ca 
effect is much earlier than two minutes as reported in this study. In addition, there is the issue of the nonsteady-state condition. While the investigators were waiting for the onset of topical lidocaine anesthetic effect, the depression of the central nervous system secondary to the induction agents was decreasing rapidly. These opposing effects cannot be ignored.

From the results of Takita's study, we can conclude that properly applied tracheal lidocaine appears to attenuate the hemodynamic responses to tracheal intubation. However, we should question the clinical significance of the hemodynamic responses associated with laryngoscopic intubation. A transient increase in arterial blood pressure and heart rate occurs in most of our daily activities and may not be of any clinical importance. In our laboratories, we have previously studied the continuous hemodynamic responses (intra-arterial blood pressure and heart rate) during a stress test in healthy volunteers $(n=21) .{ }^{10}$ During the two stages of Bruce protocol stress test (seven metabolic equivalents), the mean $( \pm$ SEM) systolic pressure increased from a baseline of $148 \pm 3 \mathrm{mmHg}$ to $185 \pm$ $2 \mathrm{mmHg}$ at six minutes, and the mean $( \pm$ SEM $)$ heart rate increased from $84 \pm 3$ beats. $\mathrm{min}^{-1}$ to $131 \pm 3$ beats. $\min ^{-1}$ at six minutes. All these hemodynamic changes returned to baseline values within four minutes of stopping activity. Under similar study conditions, we also studied hemodynamic responses in patients with stable angina $(n=19) .{ }^{11}$ The mean $( \pm$ SEM) systolic pressure and heart rate increased from a baseline of $179 \pm 7 \mathrm{mmHg}$ to $221 \pm 6 \mathrm{mmHg}$, and from $86 \pm 6$ beats. $\mathrm{min}^{-1}$ to $121 \pm 7$ beats. $\mathrm{min}^{-1}$ at $\mathrm{six}$ minutes respectively. Similarly, these hemodynamic changes returned to baseline values quickly (within four minutes) after the termination of the stress test. All the study patients completed the stress test without any difficulties. Although there was a slight ST segment depression with a mean $( \pm$ SEM) maximum depression of $-1.4 \pm 0.2 \mathrm{mV}$, all the changes resolved spontaneously during the recovery period. These stress studies serve to illustrate that hemodynamic responses occur with normal daily activities and these responses are generally well tolerated, provided they are short-lived. Certainly, the impact of these cardiovascular responses will become more profound when they are sustained for a long duration of time, particularly for patients with a severely compromised myocardial function or intravascular depletion.

In summary, in order to minimize the hemodynamic responses of tracheal intubation under anesthesia, it is important to understand the dynamic interactions between the drugs used, the onset of drug effect and the delicate balance of the opposing effects of drugs and noxious stimuli. While it is important for anesthesiologists to continue to refine anesthetic techniques in order to attenuate hemodynamic responses to tracheal intubation (and be prepared to treat unanticipated responses), one should avoid over-treating these responses which are usually short-lived and well tolerated by most patients. After all, it is much easier to avoid overdosing the patients than to treat the complications associated with an over zealous use of drugs. In other words, an ounce of prevention is worth a pound of cure.

\section{Comprendre les réac- tions hémodynamiques à l'intubation endotra- chéale}

On sait très bien que l'intubation endotrachéale laryngoscopique est associée à l'hypertension et à la tachycardie engendrées par des réactions sympathiques. Ainsi, on a suggéré de nombreuses interventions visant à atténuer ces réponses réflexes à l'intubation. Elles comprennent l'usage d'une variété d'anesthésiques, de bêtabloqueurs, de vasodilatateurs, d'anesthésiques locaux, incluant la lidocaïne et le magnésium. ${ }^{1-5}$ Malheureusement, aucune de ces manipulations pharmacologiques ne permet d'atténuer les réactions défavorables de manière constante et efficace et aucune n'est exempte de complications.

Dans un système biologique dynamique, comme le corps humain, il est souvent difficile d'appréhender la relation pharmacocinétique-pharmacodynamique complexe des effets des médicaments. Le délai d'installation de l'effet d'un anesthésique parmi les plus couramment utilisés varie de façon significative en fonction de ses caractéristiques physico-chimiques. De façon générale, à l'état d'équilibre pharmacocinétique, les relations concentration-effet peuvent être décrites par des modèles pharmacodynamiques relativement simples. ${ }^{6}$ Toutefois, la relation se complexifie lorsque le médicament n'est pas administré à l'état d'équilibre, comme c'est le cas de l'administration iv d'un bolus. Il y a alors un décalage entre les changements de concentration plasmatique des médicaments et les effets observés correspondants, et cela à cause du temps d'équilibre au site d'observation de l'effet sanguin. En conséquence, un 
modèle pharmacocinétique-pharmacodynamique plus élaboré et mieux intégré devient nécessaire pour pouvoir relier la concentration plasmatique et l'effet observé et expliquer la dissociation temporelle possible entre les deux.?

Dans des conditions de non-équilibre, les concentrations dans le plasma et au site effecteur (au cerveau, dans le cas des anesthésiques) changent rapidement à la suite de l'administration de médicament. À moins de mesures très fréquentes de l'effet médicamenteux, comme un enregistrement continu de la tension artérielle ou de l'électroencéphalogramme, il peut être impossible de caractériser pleinement la nature dynamique réelle de cet effet. Autrement dit, il est possible que l'observation de l'effet maximal nous échappe.

L'interprétation des réactions cardio-vasculaires aux anesthésiques devient encore plus difficile lorsqu'une intervention nocive, comme une intubation laryngoscopique, introduit des variables confondantes. Ces variables comprennent non seulement l'importance et la durée de l'intervention nocive, mais aussi des variables produites par diverses conditions médicales comme les maladies cardio-vasculaires et le diabète. Cette multitude de variables confondantes peut expliquer, en partie, les résultats incohérents de nombreuses études antérieures.

Dans le présent numéro, Takita et ses collègues ont vérifié l'efficacité de l'administration trachéale de lidocaïne réalisée afin de réduire les réactions cardio-vasculaires à l'intubation endotrachéale qui suit l'induction anesthésique. ${ }^{8}$ Bien que, dans le passé, on ait découvert que l'administration topique de lidocaïne était inefficace ${ }^{9}$, Takita et coll. ont adopté la démarche qui convenait en attendant le début de l'effet (deux minutes) pour permettre à l'anesthésie topique d'agir avant de réaliser l'intubation. Les réactions cardio-vasculaires chez ces patients ont été sensiblement moins importantes que chez les patients qui n'ont pas reçu de lidocaïne trachéale ou chez ceux qui ont été intubés immédiatement après l'administration de lidocaïne. Les auteurs ont conclu que la lidocaïne trachéale diminue les réactions cardio-vasculaires à l'intubation laryngoscopique, pourvu qu'elle soit administrée deux minutes avant l'intubation.

Malheureusement, les chercheurs n'ont pas utilisé de mesure de grande fréquence pendant l'évaluation du début de l'effet pharmacodynamique de la lidocaïne. Or, il se peut que le début de l'effet maximal du médicament se produise beaucoup plus tôt que deux minutes, comme on le rapporte dans cette étude. En outre, la question de l'état de non-équilibre demeure. Pendant que les expérimentateurs attendaient le début de l'effet anesthésique de la lidocaïne topique, la dépression du système nerveux central, secondaire à l'action des médicaments induisant l'anesthésie, décroissait rapidement. On ne peut passer sous silence ces effets opposés.

Nous pouvons conclure des résultats de l'étude de Takita que l'administration convenable de lidocaine trachéale semble diminuer les réactions hémodynamiques à l'intubation trachéale. Cependant, nous devons nous interroger sur l'intérêt clinique des réactions hémodynamiques associées à l'intubation. Une augmentation passagère de la tension artérielle et de la fréquence cardiaque survient dans la majorité de nos activités quotidiennes et ne sont peut-être d'aucune importance clinique. Dans nos laboratoires, nous avons déjà étudié les réactions hémodynamiques continues (tension intra-artérielle et fréquence cardiaque) pendant une épreuve d'effort chez des volontaires en bonne santé $(n=21) \cdot{ }^{10}$ Pendant les deux stades du protocole Bruce (sept équivalents métaboliques), la tension systolique moyenne ( \pm l'erreur type) a augmenté d'une mesure de base de $148 \pm 3 \mathrm{mmHg}$ à 185 $\pm 2 \mathrm{mmHg}$ à six minutes, et la fréquence cardiaque moyenne ( \pm l'erreur type) de $84 \pm 3$ battements $\times$ min $^{-1}$ à $131 \pm 3$ battements $\min ^{-1}$ à six minutes. On a retrouvé ensuite les valeurs hémodynamiques de base en moins de quatre minutes après avoir stoppé l'activité. Nous avons aussi étudié, dans des conditions similaires, les réactions hémodynamiques chez des patients atteints d'angine stable $(n=19) .{ }^{11}$ La tension systolique et la fréquence cardiaque moyennes ( \pm l'erreur type) sont passées de $179 \pm 7 \mathrm{mmHg}$ à $221 \pm 6$ $\mathrm{mmHg}$, et de $86 \pm 6$ battements $\min ^{-1}$ à $121 \pm 7$ battements $\min ^{-1}$ à six minutes, respectivement. On a encore une fois retrouvé rapidement les valeurs de base (en moins de quatre minutes) après la fin de l'épreuve d'effort. Tous les patients de l'étude ont terminé l'épreuve sans aucune difficulté. Malgré un léger abaissement du segment $S$ - $T$ et d'une baisse maximale moyenne ( \pm l'erreur type) de $-1,4 \pm 0,2 \mathrm{mV}$, tous les changements sont disparus spontanément pendant la récupération. Ces études d'effort montrent que les réactions hémodynamiques surviennent dans les activités quotidiennes normales et que ces réactions sont généralement bien tolérées pourvu qu'elles soient de courte durée. L'effet de ces réactions cardio-vasculaires deviendra certainement plus profond si l'activité est soutenue longtemps, surtout chez les patients dont la fonction myocardique est amoindrie ou en situation d'hypovolémie.

En conclusion, dans le but de réduire les réactions hémodynamiques à l'intubation endotrachéale sous anesthésie, il faut comprendre les interactions dynamiques entre les médicaments utilisés, le début de 
l'effet du médicament et l'équilibre délicat des effets opposés des médicaments et des stimuli nocifs. Quoiqu'il soit important pour les anesthésiologistes de continuer à perfectionner les techniques anesthésiques afin de réduire les réactions hémodynamiques à l'intubation endotrachéale (et d'être prêt à traiter des réactions imprévues), on devrait éviter de surtraiter ces réactions qui sont habituellement brèves et bien tolérées par la plupart des patients. Tout compte fait, il est beaucoup plus facile d'éviter les surdoses que de traiter les complications associées à un usage trop empressé de médicaments. En d'autres termes, mieux vaut prévenir que guérir.

\section{References}

l Kautto UM. Effect of combinations of topical anaesthesia, fentanyl, halothane or $\mathrm{N}_{2} \mathrm{O}$ on circulatory intubation response in normo- and hypertensive patients. Acta Anaesthesiol Scand 1983; 27: 245-51.

2 Lui PL, Gatt S, Gugino LD, Mallampati SR, Covino $B G$. Esmolol for control of increases in heart rate and blood presure during tracheal intubation after thiopentone and succinylcholine. Can Anaesth Soc J 1986; 33 : 556-62.

3 Stoelting RK. Attenuation of blood pressure response to laryngoscopy and tracheal intubation with sodium nitroprusside. Anesth Analg 1979; 58: 116-9.

4 Hamill JF, Bedford RF, Weaver DC, Colohan AR. Lidocaine before endotracheal intubation: intravenous or laryngotracheal? Anesthesiology 1981; 55: 578-81.

5 Puri GD, Marudhachalam KS, Chari P, Suri RK. The effect of magnesium sulphate on hemodynamics and its efficacy in attenuating the response to endotracheal intubation in patients with coronary artery disease. Anesth Analg 1998; 87: 808-11.

6 Meibohm B, Derendorf H. Basic concepts of pharmacokinetic/pharmacodynamic (PK/PD) modelling. Int J Clin Pharmacol Ther 1997; 35: 401-13.

7 Holford NH, Sheiner LB. Understanding the doseeffect relationship: clinical application of pharmacokinetic-pharmacodynamic models. Clin Pharmacokinet 1981; 6: 429-53.

8 Takita K, Morimoto $\Upsilon$, Kemmotsu O. Tracheal lidocaine attenuates the cardiovascular response to endotracheal intubation. Can J Anesth 2001; 48: 732-6.

9 Stoelting RK, Peterson C. Circulatory changes during anesthetic induction: impact of d-tubocurarine pretreatment, thiamylal, succinylcholine, laryngoscopy, and tracheal lidocaine. Anesth Analg 1976; 55: 77-81.

10 Yeung PK, Hung OR, Pollak PT, Klassen GA

Pharmacokinetics and hemodynamic effects of diltiazen in healthy volunteers: comparing resting with the effect of exercise. Int J Clin Pharmacol Ther 1999; 37: 413-6.
11 Klassen GA, Yeung PK, Barclay KD, Pollack PT, Hung OR, Buckley SJ. Effect of diltiazem on intraarterial blood pressure and heart rate during stress testing in patients with angina: a gender comparison study. J Clin Pharmacol 1997; 37: 297-303. 